\title{
The Psychosocial Impact of Adolescent Food Allergy: A Review of The Literature
}

Despite improvements in the treatment landscape, the psychological impact of food allergies is still yet to be fully understood. My

Editor's Pick for this year's issue is by Newman and Knibb. In this review, the authors discuss the impact of food allergies on the mental well-being of adolescents, including contributing factors to risk-taking behaviour. I hope you enjoy reading this fundamental article.
Authors:
${ }^{*}$ Kristina Newman, ${ }^{1}$ Rebecca Knibb²
1. Department of Psychology, Nottingham Trent University, Nottingham, UK
2. Department of Psychology, School of Life and Health Sciences, Aston University, Birmingham, UK
*Correspondence to knewmanpsy@gmail.com

Disclosure: $\quad$ Dr Knibb receives consultancy fees from Aimmune. Dr Newman has declared no conflicts of interest.
Acknowledgements: This work was funded by the Midlands Asthma and Allergy Research Association (MAARA).

Received:

07.04 .20

Accepted:

28.05.20

Keywords:

Adolescence, anaphylaxis, food allergy, peer relationships, psychosocial impact.

Citation:

EMJ Allergy Immunol. 2020;5[1]:54-60.

\section{Abstract}

Previous research has suggested adolescents and young people with food allergies (FA) have the highest risk of fatal reactions to food. As the prevalence of FA has been increasing there has been increased demand for psychosocial support of adolescents with food allergy, with anxiety around food and social interactions affecting the lives of adolescents and their families. This narrative review aims to explore the psychosocial impact of having a FA in adolescence, including the suggested impact, coping, risk-taking behaviour, and effect of peers and social support. The review concludes with considerations of the wider community which may also have an effect.

\section{BACKGROUND}

Food allergy (FA) is defined as an adverse reaction to ingestion of certain food types. The prevalence of FA is currently estimated at $2 \%$ of adults and $5-8 \%$ of children in the UK.' The European Academy of Allergy and Clinical Immunology (EAACl) found that cases of FA have doubled in the last decade. ${ }^{2}$ FA-related hospital admissions in the UK have increased $500 \%$ since $1990^{3}$ and seven-fold in the last decade. ${ }^{2}$ The increase in prevalence may be because of increased identification and awareness. Prevalence rates are also dependent on age and allergen, as it is expected the majority of milk and egg allergies in infants are resolved by school age whereas 
nut allergies are likely to persist throughout life, ${ }^{4}$ however any food has the potential to become an allergen. An allergy may be IgE-mediated or non-IgE-mediated; IgE are antibodies produced by the immune system that can trigger severe allergic reactions including anaphylaxis, an extreme reaction to an allergenic trigger. Reactions to FA differ by person, however, anaphylactic reactions can be fatal. Adolescents and young adults with a FA are at the highest risk of allergic reactions and mortality, ${ }^{5-7}$ and are therefore an essential population to understand and support with their condition.

There is currently no known cure for FA and management includes strict dietary observation and avoidance of possible allergens. ${ }^{8}$ If anaphylaxis occurs, an adrenaline auto-injector (AAI) should be used to control symptoms and prevent fatality ${ }^{9}$ and an ambulance should be called immediately. It is estimated that 20 people in the UK die each year from a fatal FA reaction. ${ }^{10}$

Anaphylaxis in adolescents caused by FA has received substantial media attention in the UK over the last few years, increasing awareness in its wake. In 2018, 13-year-old Karanbir Cheema had a fatal allergic reaction caused by a classmate placing cheese down their shirt whilst at school." In 2016, 15-year-old Natasha Ednan-Laperouse had a fatal allergic reaction to a take-away baguette that had an incorrectly labelled ingredient list. ${ }^{12}$ Following this, there have been campaigns to change food labelling laws. ${ }^{13}$ These adolescent fatalities show the severity of FA and the need for policy change in food establishments and schools, as well as a need to educate the public on FA to decrease the risk of fatal anaphylaxis. This narrative review therefore focusses on the breadth of the psychosocial impact of FA on adolescents and explores previous research in this area.

\section{IMPACT OF FOOD ALLERGY IN ADOLESCENCE}

DunnGalvin et al. ${ }^{14}$ suggests a direct effect on child development. At around 8 years of age, children become aware that parents are unable to ensure complete safety. This occurs in a developmental stage where children are learning to be more autonomous and find their independence as they become adolescent.
Between the ages of 8 and 12 years was considered the stage of 'growing awareness'. Through ages 13-16 years, awareness and autonomy grew further as the child adapted to new and unfamiliar contexts. ${ }^{15}$ In patients with FA, a conflict in negotiating independence and the power balance between parent and child can cause further anxiety for both. ${ }^{14}$ Knowing about these beliefs and challenges associated with having FA in childhood and adolescence may assist in understanding why adolescence is the age patients are most at risk of fatal reactions to food.

Research by DunnGalvin et al. ${ }^{14}$ has suggested that FA has an impact on psychological development and theorises that FA affects cognition, emotion, and behaviour, and that these may change and develop as children and adolescents age. Beliefs about identity, or how the participants felt about themselves, shifted at around 8 years of age. Having a food thought of as their 'special' food, as labelled by parents, marked the difference between children and adolescents. The participants felt their FA had a strong impact on who they were as a person and the lives they lived (e.g., friends, places visited, and feelings about the self). Parents, especially mothers, were considered a source of safety for those aged 6-8 years, increasing their confidence in managing the FA. This then shifted after 8 years of age when children started expressing concern over what they ate. Regarding autonomy (independence), control, and self-efficacy (control over themselves and confidence in their actions), children aged $\geq 9$ years realised parents cannot keep them safe in every situation. These feelings of increased risk were exacerbated by the belief that FA severity was not understood by the general population; symptoms were thought to be mild, which made eating out difficult. Food became an area of anxiety as children were concerned that consumption of certain foods would lead to a reaction. This had a higher impact on older participants, especially the fear of being unable to breathe, which was considered the worst symptom. Self-reported FA in the USA between 2007 and 2010 were associated with higher anxiety in adolescents, ${ }^{16}$ highlighting a population in need of further support.

Quality of life (QoL) has also been reported to be affected, in both those with FA and their families. A review by Cummings et al. ${ }^{17}$ found 
strong evidence for the impact of FA on QoL and psychosocial distress in both children and adolescents with FA and their families. FA was found to have an impact on daily life, which included disruption to family activities, concern about eating out, and a preference to continuously visit places perceived as safe, as well as patient and parent concerns about FA management in school. ${ }^{18}$ A larger number of allergic conditions (e.g., FA comorbid with eczema) have been associated with a higher report of psychosocial impact, with increasing disruption to social family activities. ${ }^{19}$ By contrast, following a negative food challenge, there was a significant improvement in the child and family's social life..$^{20}$

\section{HOW DO ADOLESCENTS WITH FOOD ALLERGY COPE WITH THEIR CONDITION?}

As living with a FA can have various psychological and social challenges, adolescents with FA may implement various coping strategies into their allergy management. Sampson et al. ${ }^{21}$ found that participants aged 13-21 years considered behavioural strategies such as carriage of their AAl and reading food labels, in addition to communication with others, as the main coping strategies in managing their FA. DunnGalvin et al. ${ }^{14}$ identified three coping strategies in FA: avoidance, minimisation, and adaptation. Avoidance strategies focus on reducing stress through avoiding the issue, for example by avoiding places associated with food. This avoidance strategy focusses on the emotions associated with risk and identity. Avoidance strategies were linked with low self-efficacy, meaning adolescents did not feel confident or in control. Avoidance strategies were also associated with high anxiety and a feeling that the FA was a big part of their identity. Cognitive minimisation strategies were more prevalent in boys who experienced bullying. This strategy involved rejection of FA as part of their identity and engagement in 'risky' actions such as not adhering to AAl carriage. By rejecting FA as part of their identity they may also reject the severity of FA, which may lead to more risky behaviour and increased risk of reaction. Finally, adaptive strategies were associated with more positive behavioural, emotional, and cognitive strategies. Adaptive strategies were more common when parents encouraged self- management and independence, which may increase the adolescents' confidence. Positive strategies include supportive peers and good communication, which were felt to be important to adolescents with FA. ${ }^{14}$

\section{ADOLESCENTS WITH FOOD ALLERGY AND RISK-TAKING BEHAVIOUR}

Adolescents and young adults with FA have the highest risk of reactions to food and the highest frequency of fatal reactions., 5 This may be attributed to increased engagement in risk-taking behaviours, ${ }^{9}$ for example not carrying an AAI. Younger children have shown less anxiety and risk-taking behaviour as they depend on parents for FA management. At around 12 years of age, conflict and resentment can arise as adolescents seek independence, ${ }^{14}$ which may be why risktaking behaviour increases.

Sampson et al. $^{9}$ suggested that risk-taking behaviour may be a core factor that results in adolescents having the highest risk of fatal anaphylactic reactions. Adolescent risk factors are thought to be influenced biologically by genetic predispositions, direct hormonal influences, asynchronous pubertal timing, and brain and central nervous system development. ${ }^{22}$ Risk-taking has also been associated with disinhibition and a risk-taking personality, and, to a lesser degree, experience-seeking, invulnerability, thrill and adventure seeking, and boredom susceptibility. ${ }^{23}$

In psychology, learnt, developmental, and personality approaches have been considered to attempt to explain risk-taking behaviour. ${ }^{23}$ The developmental approach considers that risktaking is either a normal exploratory behaviour or a negative by-product of egocentrism in cognitive development. The learnt approach considers risk-taking an act of deviance in problem behaviour, ${ }^{24}$ as a result of a poor environment, family communication, and socialisation. ${ }^{25}$ In addition, adolescents who had a personality trait of 'sensation-seekers'26 were more likely to view risk positively, especially adolescent males.

Previous research on adolescents and risk tends to focus on common coming-of-age risk-taking behaviours such as smoking, alcohol use, and unprotected sex. The more favourable an option was believed to be, the less risk was associated 
with it, ${ }^{27,28}$ which can be exacerbated by peers with similar views who may reinforce this risktaking behaviour. While some risk-taking in adolescence is to be expected as young people find their independence, in those with FA, some risk-taking behaviour can have poor health consequences. Warren et al. ${ }^{29}$ found adolescent risk-taking behaviour included eating food with 'may contain' labels, not carrying their AAI, kissing people who had recently consumed their allergen, or eating homemade or unpackaged food where they were unsure if it contained their allergen. Findings suggested that adolescents with FA were less likely to engage in risk-taking behaviours if they had a peanut allergy, overprotective mothers, teachers who were aware of their FA, supportive female friends, an established education plan, or a history of being bullied. Positive views of FA, including improved diet, empathy, and greater responsibility, also reduced risk-taking behaviour. A healthier diet may have helped the adolescents feel more positive about their FA diet restrictions, leading to heightened empathy, responsibility, and maturity.

A new potential treatment for FA may also have future effects on risk-taking behaviours. A new oral immunotherapy treatment for children aged between 4 and 7 years has been approved by the U.S. Food and Drug Administration (FDA) for the treatment of peanut allergy. ${ }^{30}$ Emergence of desensitisation treatments may affect management of FA and both reduce anxiety and increase QoL in adolescents with FA, which may, as a result, increase risk-taking behaviour. Research exploring psychological and behavioural effects of emerging oral immunotherapy treatment on adolescent FA is essential for understanding future psychosocial impact of FA.

\section{PEERS AND SOCIAL SUPPORT}

Peer relationships are an important aspect of adolescent development and can have an influence on adolescent and risk-taking behaviour in both a positive and negative way, depending on peer norms. ${ }^{30}$ Peers described as 'deviant', who engaged in delinquent or antisocial behaviour, have been linked to increased risktaking behaviour in 11-15-year olds. ${ }^{31}$
Support for adults who have FA and have experienced anaphylaxis is viewed as important, though lack of understanding can make this difficult. ${ }^{32}$ The literature on peer support for adolescents with FA is lacking, but Warren et al. ${ }^{33}$ suggested supportive female friends led to less risk-taking behaviour. Previous research suggests peer pressure may be an issue. ${ }^{9}$ This highlights that a good peer support network may have protective factors for adolescents with FA, potentially leading to fewer reactions caused by risk-taking behaviour. In the Sampson et al. ${ }^{9}$ study, greater peer education was suggested as a way to reduce teasing and bullying and improve general safety. Bullying focussed on the adolescent's FA, with acts such as throwing the food at the adolescent or threatening them with consumption, as highlighted by Stensgaard et al. ${ }^{34}$ Fenton et al. ${ }^{35}$ also found that adolescents with FA were sometimes concerned that disclosing they had a FA may affect their safety or jeopardise friendships. However, education may be difficult for adolescents with FA to deliver to their peers themselves. Peer support may help adolescents with FA to reduce risky behaviour and keep safe.

The sensitivity of social relationships with peers and parents for personal development in the adolescent period can have a negative impact on QoL in adolescents with FA. ${ }^{16,36}$ Children with FA have been reported to have worse QoL in social and psychological domains, compared to parents. ${ }^{36}$ Social limitations in FA have been highlighted in children of various ages, including playing at friend's houses, sleepovers, parties, field trips, and also in family social events. ${ }^{37}$ This highlights the importance of building strong relationships with peers and family to reduce social limitations for adolescents with FA.

\section{SCHOOLS}

A school environment can also pose a risk for someone with FA. Over 25\% of European school children have an allergy and $20 \%$ of FA reactions occur in schools. ${ }^{38}$ Furthermore, up to two-thirds of schools have a minimum of one child at risk of anaphylaxis, and may not be suitably prepared in the event of a reaction. ${ }^{38}$ Food Allergy Research and Education (FARE) report that 15\% of American school-aged children with FA have had an allergic reaction in school. ${ }^{39}$ Previous 
research highlights that schools are the most common location for anaphylaxis. ${ }^{7,40,41}$ All schools should have a protective policy in place for their students with FA, however many of these policies could be improved. Personalised emergency management plans that detail individual reaction symptoms and a plan of action for when they occur are not consistently provided ${ }^{41}$ and teachers are reported to have poor knowledge about anaphylaxis, including symptoms, triggers, and AAl. ${ }^{42,43}$ However, much of this research was carried out in America, and therefore there may be differences when compared to other countries.

In Canada, adolescents reported that the transition to secondary school was difficult as schools were larger and less organised, which made them feel less safe. ${ }^{44}$ School policies could also be exclusionary, where adolescents with FA had to sit alone at lunch or were not allowed to attend school trips. ${ }^{43,45}$ Finding their FA embarrassing or shameful can create issues in the school environment, especially if peers are unaware of the $\mathrm{FA},{ }^{9}$ as adolescents with FA may be secretive to protect themselves or avoid unwanted attention. School trips have also been described as difficult and annoying if the adolescent with FA could not eat "the same as everyone else." 33 When schools were accommodating of FA, children felt safer and more included, ${ }^{45}$ however, this also drew attention to how they were different from their peers. ${ }^{44}$ Understanding peer beliefs may assist with these issues by suitable school-based interventions.

\section{WIDER COMMUNITY}

Adolescents with FA may experience a reaction in a variety of community settings such as restaurants, beaches, sports fields, or gymnasiums, ${ }^{40}$ as well as potential fatalities in restaurants, a friend's home, or work. ${ }^{10}$ Allergen avoidance training often considers avoidance of allergens in the home, but focusses less on how to avoid exposure to allergens in the community. ${ }^{46}$

Knowledge and attitudes of the community can be a barrier for those with FA, particularly in those who directly interact with consumer food. Confusion has been reported in UK takeaway staff, ${ }^{47}$ who were unsure of the difference between milk allergy and lactose intolerance and whether allergens could be transferred by hands. A USA study on restaurant workers showed that very few knew how to assist someone experiencing anaphylaxis and staff were not ready to manage FA safely. ${ }^{48}$ These attitudes were similar in European countries such as Germany. ${ }^{49}$ Further research into food handlers ${ }^{50,51}$ also found knowledge could be improved, and suggested that allergy knowledge was significantly correlated with practice, confidence, and care of workers.

Improved FA knowledge of the general public would be beneficial, is desirable, ${ }^{46}$ and must accommodate the general public's needs whilst balancing protection of those with FA. This is an important concern in the UK as well as in other developed countries such as the USA, Canada, Australia, and New Zealand.52 However, some policies have already been met with resistance, such as nut bans in schools. ${ }^{53}$ Nut bans in schools are claimed to be extreme and limiting of food choice by those without FA, as documented in the Canadian study by Harrington et al. ${ }^{54}$ In this study, peanut bans faced backlash as peanut butter is an accessible and affordable source of protein in low-income families. In the UK, advice from charities such as Anaphylaxis Campaign ${ }^{55}$ is not to use allergen bans, as there is no guarantee it could be a safe environment as children can accidently bring in the banned food from home. This can also create conflict between parents, which may actually increase risk of reaction. As conflict and social exclusion are concerns for those with FA, community interventions should be conducted delicately. ${ }^{46}$ As there are different governing bodies for health and education, cooperation between healthcare professionals such as doctors and dietitians, the school community, and parents are necessary to overcome these barriers. ${ }^{46,56}$ Community interventions may involve addressing misinformation, providing education, and addressing FA condition beliefs, for example that FA is not a serious condition. Jones et al. ${ }^{57}$ found that support groups for young people with FA resulted in improved self-esteem and confidence, both generally and when managing their FA. These groups helped adolescents feel included and share experiences with people who also had FA, which was highly valued. Furthermore, support groups and anaphylaxis management plans were associated with good 
adherence to self-care behaviours, ${ }^{58}$ leading the authors to suggest these supportive and applied planning elements may be more useful in related interventions rather than interventions focussing on increasing knowledge.

\section{CONCLUSION}

FA is a condition of interest and concern because of its rising prevalence, increasing reports of psychological distress, and increasing parental conflict regarding developing independence in adolescence. Adolescence is the age group most at risk of anaphylactic reactions, but it is not yet fully understood why. Understanding the experiences and feelings of adolescents about FA is important to give insight into how adolescents both with and without FA think and feel about the condition. This may also provide suggestions on how to reduce risk-taking behaviour, improve adherence to AAl carriage, and reduce the likelihood of anaphylactic reactions. This may also identify areas where adolescents need more support; for example, in managing their FA as they become more independent and keeping beliefs realistic rather than destructive, such as unrealistic optimism (believing a reaction will never happen to them) or the belief they are surrounded by danger, leading to high levels of anxiety.

\section{References}

1. Food Standards Agency (FSA). Food Standards Agency: Board Meeting 15 March 2017: Food allergy and intolerance programme. 2017. Available at: https://www.food.gov.uk/ sites/default/files/media/document/ fsa170306.pdf. Last accessed: 30 January 2020.

2. European Academy of Allergy and Clinical Immunology (EAACI). Food Allergy \& Anaphylaxis Public Declaration. 2015. Available at: http://www. eaaci.org/attachments/FoodAllergy \&AnaphylaxisPublicDeclarationCombined.pdf. Last accessed: 30 January 2020.

3. Gupta R et al. Time trends in allergic disorders in the UK. Thorax. 2007;62(1):91-6.

4. Sicherer SH, Sampson HA. Food allergy: epidemiology, pathogenesis, diagnosis, and treatment. J Allergy Clin Immunol. 2014;133(2):291-307.e5.

5. Bock SA et al. Further fatalities caused by anaphylactic reactions to food, 2001-2006. J Allergy Clin Immunol. 2007;119(4):1016-8.

6. Pumphrey RSH. Lessons for management of anaphylaxis from a study of fatal reactions. Clin Exp Allergy. 2000;30(8):1144-50.

7. Conroy ME. Food allergy: review of epidemiology, risk factors, and future treatments. AM J Lifestyle Med. 2013;9(4):280-7.

8. Sheikh A, Alves B. Hospital admissions for acute anaphylaxis: time trend study. BMJ. 2000;320:1441.

9. Sampson MA et al. Risk-taking and coping strategies of adolescents and young adults with food allergy. J Allergy Clin Immunol. 2006;117(6):1440-5.

10. Pumphrey RSH, Gowland $\mathrm{MH}$.
Further fatal allergic reactions to food in the United Kingdom, 1999-2006. J Allergy Clin Immunol. 2007;119(4):1018-9.

11. Telegraph. Boy, 13, with dairy allergy died after schoolmate threw cheese down his T-shirt, inquest hears. 2019. Available at: https://www.telegraph. co.uk/news/2018/09/19/boy-13-dairyallergy-died-schoolmate-threwcheese-t-shirt-inquest/\#: :text=A\%20 boy\%20with\%20a\%20severe,on\%20 June\%2028\%20last\%20year. Last accessed: 4 December 2019.

12. BBC News. Food allergies: tougher labelling law to prevent deaths. 2019. Available at: https://www.bbc co.uk/news/health-46994179. Last accessed: 4 December 2019.

13. GOV.UK. Gove to introduce 'Natasha's Law.' 2019. Available at: https:// www.gov.uk/government/news/ gove-to-introduce-natashas-law. Last accessed: 16 December 2019

14. DunnGalvin A et al. Developmental pathways in food allergy: a new theoretical framework. Allergy. 2009;64(4):560-8.

15. DunnGalvin A et al. Preliminary development of the food allergy coping and emotions questionnaires for children, adolescents, and young people: qualitative analysis of data on IgE-mediated food allergy from five countries. J Allergy Clin Immunol Pract. 2018;6(2):506-13.

16. Dantzer J, Keet CA. Anxiety associated with food allergy adults and adolescents: an analysis of data from the NHANES 20072020. J Allergy Clin Immunol. 2020;8(15):1743-6.

17. Cummings $A$ et al. The psychosocial impact of food allergy on children and adolescents: a review. Allergy.

\section{0;65(8):933-45.}

18. Valentine T, Knibb RC. Exploring quality of life in families of children with and without a severe food allergy. Appetite. 2011;57(2):467-74.

19. Marklund B. Health-related quality of life among adolescents with allergylike conditions - with emphasis on food hypersensitivity. Health Qual Life Outcomes. 2004;2:65.

20. Eigenmann PA et al. Continuing foodavoidance diets after negative food challenges. Pediatr Allergy Immunol. 2006;17(8):601-5.

21. Sampson MA et al. Risk-taking and coping strategies of adolescents and young adults with food allergy. J Allergy Clin Immunol. 2006;117(6):1440-5.

22. Sales JM, Irwin CE, "a biopsychosocial perspective of adolescent health and disease," O'Donohue W et al. (eds.), Handbook of Adolescent Health Psychology (2013), Springer: New York, NY, pp.13-29.

23. Greene $\mathrm{K}$ et al. Targeting adolescent risk-taking behaviours: the contributions of egocentrism and sensation seeking. J Adolesc. 2000;23(4):439-61.

24. Donovan JE, Jessor R. Structure of problem behaviour in adolescence and young adulthood. J Consult Clin Psychol. 1985;53(6):890-904.

25. Gore S, Eckenrode J, "Context and processes in research on risk and resilience," Haggery RJ et al. (eds.) Stress, Risk and Resilience in Children and Adolescents: Processes, Mechanisms and Interventions (1994). New York: Cambridge University Press, pp.19-57.

26. Zuckerman M, Neeb M. Sensation seeking and psychopathology. 
Psychiatry Res. 1979;1(3):255-64.

27. Romer D. Adolescent risk taking impulsivity, and brain development: Implications for prevention. Dev Psychobiol. 2010;52(3):263-76.

28. Allen JP et al. The two faces of adolescents' success with peers: adolescent popularity, social adaptation, and deviant behavior. Child Dev. 2005;76(3):747-60.

29. Warren CM et al. Food allergyrelated risk-taking and management behaviors among adolescents and young adults. J Allergy Clin Immunol Pract. 2016;5(2):381-90.

30. U.S. Food and Drug Administration (FDA). FDA approves first drug for treatment of peanut allergy for children. 2020. Available at: https:// www.fda.gov/news-events/pressannouncements/fda-approves-firstdrug-treatment-peanut-allergychildren. Last accessed: 18 May 2020.

31. Ary DV. Adolescent problem behavior: the influence of parents and peers. Behav Res Ther. 1999;37(3):21730.

32. Knibb CJ. "It's not an illness, it's just bad luck": the impact of anaphylaxis on quality of life in adults. Clin Exp Allergy. 2019;49:1040-6.

33. Warren CM. Food allergy-related risktaking and management behaviors among adolescents and young adults. J Allergy Clin Immunol Pract. 2016;5(2):381-90.

34. Stensgaard A et al. Peanut allergy as a family project: social relations and transitions in adolescence. $\mathrm{J} \mathrm{Clin}$ Nurs. 2017:26(21-22):3371-81.

35. Fenton NE et al. Tag, you're different: the interrupted spaces of children at risk of anaphylaxis. Child Geogr. 2013;11(3):281-97.

36. Morou $Z$ et al. Health related quality of life in children with food allergy and their parents: A systematic review of the literature. $\mathrm{J}$ Investig Allergol Clin Immunol. 2014;24(6):382-95.

37. Bollinger ME et al. The impact of food allergy on the daily activities of children and their families. Ann Allerg Asthma Immunol. 2006;96(3):415-21.

38. Muraro A et al. The management of the allergic child at school: EAACl/ GA2LEN Task Force on the allergic child at school. Allergy. 2010;65:681-9.

39. Food Allergy Research and Education. Facts and statistics. 2020. Available at: https://www.foodallergy. org/resources/facts-and-statistics. Last accessed: 24 May 2020.

40. Mclntyre $\mathrm{CL}$ et al. Administration of epinephrine for life-threatening allergic reactions in school settings. Pediatrics. 2005;116(5):1134-40.

41. Young $M C$ et al. Management of food allergies in schools: a perspective for allergists. J Allergy Clin Immunol. 2009;124(2):175-82.e4.

42. Ercan $\mathrm{H}$ et al. Primary school teachers' knowledge about and attitudes toward anaphylaxis. Pediatr Allergy Immunol. 2012;23(5):428-32.

43. Fenton NE et al. Illustrating risk: anaphylaxis through the eyes of the food-allergic child. Risk Anal. 2011:13(1):171-83.

44. Dean J et al. Disclosing food allergy status in schools: health-related stigma among school children in Ontario. Health Soc Care Community. 2016:24(5):43-52.

45. Stjerna ML. Food, risk and place: agency and negotiations of young people with food allergy. Sociol Health IIIn. 2015;37(2):284-97.

46. Muraro $A$ et al. EAACl food allergy and anaphylaxis guidelines: managing patients with food allergy in the community. Allergy. 2014;69(8):104657.

47. Soon JM. 'No nuts please': food allergen management in takeaways' Food Control. 2018;91:349-56.

48. Dupuis $R$ et al. Food allergy management among restaurant workers in a large U.S. city. Food Control. 2016;63:147-57.

49. Loerbroks A et al. Food allergy knowledge, attitudes and their determinants among restaurant staff: a cross-sectional study. PLoS One. 2019;14(4):e0214625.

50. Shafie AA, Azman AW. Assessment of knowledge, attitude and practice of food allergies among food handlers in the state of Penang, Malaysia. Public Health. 2015;129(9):1278-84.

51. Choi JH, Rajagopal L. Food allergy knowledge, attitudes, practices, and training of foodservice workers at a university foodservice operation in the Midwestern United States. Food Control. 2012;31(2):474-81.

52. Chafen JJS et al. Diagnosing and managing common food allergies. J JAMA. 2010;303(18):1848-56.

53. Gupta RS et al. Food allergy knowledge, attitudes, and beliefs of parents with food-allergic children in the United States. Pediatr Allergy Immunol. 2010;21(6):927-34.

54. Harrington DW et al. Exploring the determinants of the perceived risk of food allergies in Canada. Hum Ecol Risk Assess. 2012;18(6):1338-58.

55. Anaphylaxis Campaign. The Anaphylaxis Campaign's views on the proposed changes to School Foods Standards, to be made statutory from January 2015. 2015. Available at: https://www. anaphylaxis.org.uk/2015/07/20/ the-anaphylaxis-campaigns-viewson-the-proposed-changes-toschool-foods-standards-to-be-madestatutory-from-january-2015/. Last accessed: 04 January 2020.

56. Behrmann J. Ethical principles as a guide in implementing policies for the management of food allergies in schools. J Sch Nurs. 2010;26(3):18393

57. Jones $\mathrm{CJ}$ et al. Exploring what motivates and sustains support group engagement amongst young people with allergies: a qualitative study. Clin Exp Allergy. 2018;48(9):1195-205.

58. Jones $\mathrm{CJ}$ et al. Factors associated with good adherence to self-care behaviours amongst adolescents with food allergy. Pediatr Allergy Immunol. 2015:26(2):111-8. 